\title{
Convergence properties \\ for a generalization of the Caginalp phase field system*
}

\author{
Giacomo Canevari and Pierluigi Colli \\ Dipartimento di Matematica "F. Casorati", Università di Pavia \\ Via Ferrata, 1, 27100 Pavia, Italy \\ E-mail: giacomo.canevari@gmail.com pierluigi.colli@unipv.it
}

\begin{abstract}
We are concerned with a phase field system consisting of two partial differential equations in terms of the variables thermal displacement, that is basically the time integration of temperature, and phase parameter. The system is a generalization of the well-known Caginalp model for phase transitions, when including a diffusive term for the thermal displacement in the balance equation and when dealing with an arbitrary maximal monotone graph, along with a smooth anti-monotone function, in the phase equation. A Cauchy-Neumann problem has been studied for such a system in [7], by proving well-posedness and regularity results, as well as convergence of the problem as the coefficient of the diffusive term for the thermal displacement tends to zero. The aim of this contribution is rather to investigate the asymptotic behaviour of the problem as the coefficient in front of the Laplacian of the temperature goes to 0: this analysis is motivated by the types III and II cases in the thermomechanical theory of Green and Naghdi. Under minimal assumptions on the data of the problems, we show a convergence result. Then, with the help of uniform regularity estimates, we discuss the rate of convergence for the difference of the solutions in suitable norms.
\end{abstract}

Key words: phase field model, initial-boundary value problem, regularity of solutions, convergence, error estimates.

AMS (MOS) Subject Classification: 35G61, 35B30, 35B40, 80A22.

\section{Introduction}

In this paper we consider the initial and boundary value problem

$$
\begin{array}{cc}
w_{t t}-\alpha \Delta w_{t}-\beta \Delta w+u_{t}=f & \text { in } \Omega \times(0, T) \\
u_{t}-\Delta u+\gamma(u)+\bar{g}(u) \ni w_{t} & \text { in } \Omega \times(0, T)
\end{array}
$$

*Acknowledgment. The financial support of the MIUR-PRIN Grant 2008ZKHAHN "Phase transitions, hysteresis and multiscaling" and of the IMATI of CNR in Pavia is gratefully acknowledged. 


$$
\begin{gathered}
\partial_{n} w=\partial_{n} u=0 \quad \text { on } \Gamma \times(0, T) \\
w(\cdot, 0)=w_{0}, \quad w_{t}(\cdot, 0)=v_{0}, \quad u(\cdot, 0)=u_{0} \quad \text { in } \Omega
\end{gathered}
$$

where $\Omega \subset \mathbb{R}^{3}$ is a bounded domain with smooth boundary $\Gamma, T>0$ represents some finite time, and $\partial_{n}$ denotes the outward normal derivative on $\Gamma$. Moreover, $f$ is a given source term in equation (1.1), $\bar{g}: \mathbb{R} \rightarrow \mathbb{R}$ is a Lipschitz-continuous function, and $\gamma: \mathbb{R} \rightarrow 2^{\mathbb{R}}$ is a maximal monotone graph (cf., e.g., [4] or [3]): as $\gamma$ can be multivalued, in (1.2) there is the inclusion instead of the equality. Finally, $w_{0}, v_{0}, u_{0}$ stand for initial data.

Problem (1.1) -(1.4) has been studied in the paper [7] when $\alpha$ and $\beta$ are two positive coefficients; also, still in [7] the asymptotic behaviour of the problem as $\beta \searrow 0$ has been investigated. The interest of this new contribution is to examine the asymptotic properties of (1.1)-(1.4) as $\alpha \searrow 0$, being $\beta>0$ fixed once and for all. Indeed, both asymptotic investigations are interesting and deserve to be investigated, in such a way that the results proved in this paper give a completion to those of [7].

Let us point out that the coupled equations (1.1)-(1.2) form a system of phase field type. From the seminal work of Caginalp and coworkers (see, e.g., [5, 6]) it became clear that phase field systems are particularly suited to represent the dynamics of moving interfaces arising in thermally induced phase transitions. In our case, we consider the following expression for the total free energy

$$
\Psi(\theta, u)=\int_{\Omega}\left(-\frac{1}{2} \theta^{2}-\theta u+\phi(u)+G(u)+\frac{1}{2}|\nabla u|^{2}\right)
$$

where the variables $\theta$ and $u$ denote the (relative) temperature and the order parameter, respectively. We remark at once that our $w$ represents the thermal displacement variable, related to $\theta$ by

$$
w(\cdot, t)=w_{0}+(1 * \theta)(\cdot, t)=w_{0}+\int_{0}^{t} \theta(\cdot, s) d s, \quad t \in[0, T] .
$$

The integrand in (1.5) is characterized by the presence of different terms: the first one yields the concave purely caloric contribution, the second is the only term coupling the two variables, $\phi+G$ is a function acting on $u$ and the last one is accounting for surface effects. The convex and lower semicontinuous function $\phi:[0,+\infty] \rightarrow \mathbb{R}$ satisfies $\phi(0)=0=\min \phi$ and its subdifferential $\partial \phi$ coincides with $\gamma$, while $G$ stands for a smooth, in general concave, function such that $G^{\prime}=\bar{g}$. A typical example for $\phi(u)+G(u)$ is offered by the double obstacle case, in which

$$
\phi(u)=I_{[-1,+1]}(u)=\left\{\begin{array}{ll}
0 & \text { if }|u| \leq 1 \\
+\infty & \text { if }|u|>1
\end{array}, \quad G(u)=1-u^{2}\right.
$$

the two wells of $\phi+G$ being located in -1 and +1 : actually, one of the two is preferred as minimum of the potential in (1.5), according to whether the temperature $\theta$ is negative or positive. The above example perfectly fits with the systematic view and designs of Michel Frémond in non-smooth Thermomechanics [12]. In the case of (1.7), the subdifferential of the indicator function of the interval $[-1,+1]$ reads

$$
\xi \in \partial I_{[-1,+1]}(u) \quad \text { if and only if } \quad \xi\left\{\begin{array}{lll}
\leq 0 & \text { if } u=-1 \\
=0 & \text { if } & |u|<1 \\
\geq 0 & \text { if } & u=+1
\end{array}\right.
$$


so that here $\gamma=\partial I_{[-1,+1]}$ is really a graph with vertical lines. Let us also note that even a multi-well potential may be considered in (1.5): indeed, it turns out that the function $u \mapsto-\theta u+\phi(u)+G(u)$ can exhibit different wells according especially to the shape of $G$ in the region where $\phi \neq+\infty$.

A different word used for the variable $w$ in (1.6) is "freezing index": this terminology is motivated by earlier studies on the Stefan problem [11, 1] and preferred by some authors (cf. [17, 12]). We notice that a meaningful variable of the Stefan problem is the enthalpy $e$, which is defined as minus the variational derivative of $\Psi$ with respect to $\theta$ :

$$
e=-d_{\theta} \Psi, \quad \text { whence } e=\theta+u=w_{t}+u .
$$

Then, the governing balance and phase equations are given by

$$
\begin{array}{r}
e_{t}+\operatorname{div} \mathbf{q}=f \\
u_{t}+d_{u} \Psi=0
\end{array}
$$

where $\mathbf{q}$ denotes the thermal flux vector and $d_{u} \Psi$ stands for the variational derivative of $\Psi$ with respect to $u$. Hence, (1.10) reduces exactly to (1.2) along with the Neumann homogeneous boundary condition for $u$. On the other hand, if we assume the classical Fourier law

$$
\mathbf{q}=-\nabla \theta=-\nabla w_{t}
$$

(for the moment let us take the heat conductivity coefficient just equal to 1 ), then (1.9) is nothing but the usual energy balance equation as in the Caginalp model [5]. One obtains the same equation as in the weak formulation of the Stefan problem, in which however (1.2) is replaced by the mere pointwise inclusion $u \in\left(\partial I_{[-1,+1]}\right)^{-1}(\theta)$, that equivalently reads $\partial I_{[-1,+1]}(u) \ni w_{t}$.

An alternative approach to the Fourier diffusive law consists in adopting the so-called Cattaneo-Maxwell law (see, e.g., [8, 10, 18] and references therein) in which a time derivative of the flux appears:

$$
\mathbf{q}+\varepsilon \mathbf{q}_{t}=-\nabla \theta, \quad \text { for } \varepsilon>0 \text { small. }
$$

The Cattaneo-Maxwell law leads to the following equation

$$
\varepsilon \theta_{t t}+\theta_{t}-\Delta \theta+u_{t}+\varepsilon u_{t t}=f+\varepsilon f_{t} \quad \text { in } \Omega \times(0, T),
$$

which has been investigated in [18, 22]. On the other hand, solving (1.11) with respect to $\mathbf{q}$ yields

$$
\mathbf{q}=\mathbf{q}_{\mathbf{0}}+k_{\varepsilon} * \nabla \theta, \quad \text { where }\left(k_{\varepsilon} * \nabla \theta\right)(x, t):=\int_{0}^{t} k_{\varepsilon}(t-s) \nabla \theta(x, s) d s
$$

$\mathbf{q}_{\mathbf{0}}(x, t)$ is known and can be incorporated in the source term, while $k_{\varepsilon}(t)$ is a given kernel depending on $\varepsilon$. Then, from (1.9) and a prescription like $\mathbf{q}=\mathbf{q}_{0}+k * \nabla \theta$ for some fixed kernel $k$, we obtain the balance equation for the standard phase field model with memory which has a hyperbolic character and has been extensively studied in [8, 9].

Green and Naghdi (see [13, 14, 15, 16]) presented an alternative treatment for a thermomechanical theory of deformable media. This theory takes advantage of an entropy 
balance rather than the usual entropy inequality. If we restrict our attention to the heat conduction, these authors proposed three different hypotheses, labeled as type I, type II and type III, respectively. In particular, when type I is linearized, we recover the classical Fourier law (in terms of thermal displacement)

$$
\mathbf{q}=-\alpha \nabla w_{t}, \quad \alpha>0 .
$$

Type II is characterized by

$$
\mathbf{q}=-\beta \nabla w, \quad \beta>0 .
$$

Moreover, a linearized version of type III reads

$$
\mathbf{q}=-\alpha \nabla w_{t}-\beta \nabla w .
$$

We point out that (1.13) -(1.14) laws have been recently discussed, applied and compared by Miranville and Quintanilla in [19, 20, 21] (there the reader can find a rich list of references as well). In particular, our equation (1.1) reflects the use of (1.14), along with (1.9) and (1.8), in deriving it; further, a no flux boundary condition for $\mathbf{q}$ yields $\partial_{n} w=0$ in $(1.3)$.

Here we are: the system (1.1)-(1.4) comes as a consequence of (1.9)-(1.10) when (1.5) and (1.14) are postulated. Existence, continuous dependence, and regularity of the solution to the initial-boundary value problem (1.1)-(1.4) have been studied in [7]. Observe that $\gamma$ is an arbitrary maximal monotone graph, therefore $\gamma$ may be multivalued, singular and with bounded domain. To this concern, in addition to the example (1.7) we also mention the singular case investigated in [21] for a convex potential $\phi$ with the same interval $[-1,+1]$ as domain, but with

$$
\phi(u)=\kappa_{1}((1+u) \ln (1+u)+(1-u) \ln (1-u)),
$$

and for $\bar{g}(u)=G^{\prime}(u)=-2 \kappa_{0} u$, where the meaningful constants $\kappa_{0}, \kappa_{1}$ have to satisfy $0<\kappa_{1}<\kappa_{0}$.

An interesting issue is of course the investigation of the asymptotic behaviour of the solutions to problem (1.1)-(1.4) as one of the two parameters $\alpha$ or $\beta$ tends to 0. In [7] we have examined the limiting case $\beta \searrow 0$, keeping $\alpha>0$ fixed and obtaining convergence of solutions to the analogous problem with $\beta=0$, which corresponds to (1.12), i.e., the type I case of Green and Naghdi. The paper [7] also deals with two error estimates of the difference of solutions in suitable norms, showing a rate of convergence that is linear with respect to $\beta$ in both estimates.

When discussing the limit with $\alpha>0$ fixed, one can always exploit the properties of the parabolic term $-\alpha \Delta w_{t}$. This is no longer possible in the study of the limit as $\alpha \searrow 0$, which is the objective of the present paper. Indeed, if we let $\alpha \searrow 0$ and hold $\beta>0$ fixed, we are led to the analog of problem (1.1) -(1.4) with $\alpha=0$, in which equation (1.1) becomes hyperbolic. This limiting situation gives account of the type II hypothesis (1.13) of Green and Naghdi.

A change of variable turns out to be useful in obtaining estimates which does not depend on $\alpha$. Let $y$ denote the time-integrated enthalpy (cf. (1.8))

$$
y=w+1 * u .
$$


Then, problem (1.1)-(1.4) appears to be equivalent, in terms of $(y, u)$, to the problem

$$
\begin{gathered}
y_{t t}-\alpha \Delta y_{t}-\beta \Delta y=-\alpha \Delta u-\beta \Delta(1 * u)+f \quad \text { in } \Omega \times(0, T) \\
u_{t}-\Delta u+\gamma(u)+g(u) \ni y_{t} \quad \text { in } \Omega \times(0, T) \\
\partial_{n} y=\partial_{n} u=0 \quad \text { on } \Gamma \times(0, T) \\
y(\cdot, 0)=w_{0}, \quad y_{t}(\cdot, 0)=v_{0}+u_{0}, \quad u(\cdot, 0)=u_{0} \quad \text { in } \Omega,
\end{gathered}
$$

where the function $g$ is defined by $g(s)=\bar{g}(s)+s$ for all $s \in \mathbb{R}$ and results Lipschitzcontinuous whenever $\bar{g}$ is. Let us notice that in the case $\alpha=0$ problem (1.15)-(1.18) reduces to a system for which well-posedness and regularity results have been proved in $[8,9]$.

By this change of variables, and dealing with some technicalities, we are able to provide a priori estimates on the solution of (1.15)-(1.18), independent of $\alpha$, which allow us to state a convergence result as $\alpha \searrow 0$ and prove it via compactness arguments. Then we investigate the bahaviour of the difference of the solutions to the problems with $\alpha>0$ and $\alpha=0$ : a first estimate on the convergence rate is obtained, although it is not linear but of order $1 / 2$ in $\alpha$. Stronger regularity estimates independent of $\alpha$ are then provided, and they enable us to produce also a linear estimate for the convergence rate, but only in the case when $\gamma$ is a smooth single-valued function defined on the whole real line. This assumption, although limitative, is not unrealistic, because it covers the physically relevant case of the well-known 'double-well' potential [5] given by

$$
\phi(u)+G(u)=\frac{\kappa}{4}\left(u^{2}-1\right)^{2}, \quad \text { so that } \gamma(u)=\kappa u^{3} \text { and } g(u)=(1-\kappa) u,
$$

for some positive coefficient $\kappa$.

The paper is organized as follows. In Section 2 we present the main results related to the problem: the existence and uniqueness of a weak solution for both cases $\alpha>0$ and $\alpha=0$, the convergence theorem as $\alpha \searrow 0$, the regularity results yielding a strong solution and the first error estimate, the further regularity properties on the phase variable $u$ and the enthalpy $y_{t}$, and finally the second error estimate. Next, in Section 3 we recall a useful lemma for parabolic problems and then prove the convergence result. Strong solutions and the first estimate on the order of convergence are discussed in Section 4 . The concluding Section 5 is devoted to the case of a smooth potential $\phi$ and it contains the proofs of the additional regularity estimates and of the linear rate of convergence for the difference of solutions in suitable norms.

\section{Notation and main results}

Before stating clearly the formulation of the problem and the main results we achieve, we recall some notation. Let $\Omega \subseteq \mathbb{R}^{3}$ be a bounded smooth domain, with boundary $\Gamma=\partial \Omega$, and let $T>0$ be some final time. Set

$$
\begin{gathered}
Q_{t}=\Omega \times(0, t), \quad Q=Q_{T}, \\
H=L^{2}(\Omega), \quad V=H^{1}(\Omega), \quad W=\left\{v \in H^{2}(\Omega): \partial_{n} v=0 \text { a.e. on } \Gamma\right\} .
\end{gathered}
$$


We embed $H$ in $V^{\prime}$, by means of the $L^{2}(\Omega)$ inner product:

$$
\langle h, v\rangle=(h, v)_{H} \quad \text { for all } h \in H, v \in V \text {; }
$$

we warn that $\|\cdot\|_{H}$ means the norm of both $H$ and $H^{3}$. If $a$ is a function of space and time variables, we define the function $1 * a$ by the formula

$$
(1 * a)(t)=\int_{0}^{t} a(s) d s, \quad 0 \leq t \leq T
$$

The symbol $c$ stands for different positive constants, depending on $\Omega, T$ and the data, which stay bounded whenever $\alpha, \beta$ vary in a bounded subset of $(0,+\infty)$. In particular, for the sake of simplicity let us assume that

$$
0<\alpha \leq 1
$$

Any positive constant depending on the $\Omega, T$ and the data, which may tend to $+\infty$ when $\beta \searrow 0$ but stays bounded as $\alpha \searrow 0$, will be denoted by $c_{\beta}$.

For the sake of clarity, we call $\mathcal{D}_{\alpha}=\left\{w_{0, \alpha}, v_{0, \alpha}, u_{0, \alpha}, f_{\alpha}\right\},\left(y_{\alpha}, u_{\alpha}, \xi_{\alpha}\right)$ the family of data and the solution of the problem (1.15)-(1.18) (cf. also (1.1)-(1.4)), while $\mathcal{D}=$ $\left\{w_{0}, u_{0}, v_{0}, f\right\}$ and $(y, u, \xi)$ will denote the variables of related problem with $\alpha=0$.

Now we deal with the assumptions on data. We require that

$$
0<\alpha \leq 1, \quad \beta>0
$$

$\gamma \subseteq \mathbb{R} \times \mathbb{R}$ maximal monotone graph, with $\gamma(0) \ni 0$

$\phi: \mathbb{R} \longrightarrow[0,+\infty]$ convex and lower semicontinuous, with $\phi(0)=0$ and $\partial \phi=\gamma$

$$
g: \mathbb{R} \longrightarrow \mathbb{R} \text { Lipschitz-continuous }
$$

$$
f_{\alpha} \in W^{1,1}\left(0, T ; V^{\prime}\right)+L^{1}(0, T ; H)
$$

$$
w_{0, \alpha} \in V, \quad v_{0, \alpha} \in H, \quad u_{0, \alpha} \in H, \quad \phi\left(u_{0, \alpha}\right) \in L^{1}(\Omega) ;
$$

we also assume, for the norms of the data, a bound independent of $\alpha$ :

$$
\left\|f_{\alpha}\right\|_{W^{1,1}\left(0, T ; V^{\prime}\right)+L^{1}(0, T ; H)}+\left\|w_{0, \alpha}\right\|_{V}+\left\|v_{0, \alpha}\right\|_{H}+\left\|u_{0, \alpha}\right\|_{H}+\left\|\phi\left(u_{0, \alpha}\right)\right\|_{L^{1}(\Omega)} \leq c .
$$

We denote the effective domain of $\gamma$ with $D(\gamma)$. Now, we are ready to formulate precisely the problem as subsequent to the change of variable.

Problem $\left(\mathbf{P}_{\alpha, \beta}\right)$. Find $\left(y_{\alpha}, u_{\alpha}, \xi_{\alpha}\right)$ satisfying

$$
\begin{gathered}
y_{\alpha} \in W^{2,1}\left(0, T ; V^{\prime}\right) \cap W^{1, \infty}(0, T ; H) \cap L^{\infty}(0, T ; V) \\
u_{\alpha} \in H^{1}\left(0, T ; V^{\prime}\right) \cap C^{0}([0, T] ; H) \cap L^{2}(0, T ; V) \\
\xi_{\alpha} \in L^{2}(Q), \quad u_{\alpha} \in D(\gamma) \text { and } \xi_{\alpha} \in \gamma\left(u_{\alpha}\right) \text { a.e. in } Q \\
\left\langle\partial_{t}^{2} y_{\alpha}(t), v\right\rangle+\alpha\left(\nabla \partial_{t} y_{\alpha}(t), \nabla v\right)_{H}+\beta\left(\nabla y_{\alpha}(t), \nabla v\right)_{H}=\alpha\left(\nabla u_{\alpha}(t), \nabla v\right)_{H} \\
+\beta\left(\nabla\left(1 * u_{\alpha}\right)(t), \nabla v\right)_{H}+\left\langle f_{\alpha}(t), v\right\rangle \quad \text { for all } v \in V \text { and a.a. } t \in(0, T)
\end{gathered}
$$




$$
\begin{array}{r}
\left\langle\partial_{t} u_{\alpha}(t), v\right\rangle+\left(\nabla u_{\alpha}(t), \nabla v\right)_{H}+\left(\xi_{\alpha}(t), v\right)_{H}+\left(g\left(u_{\alpha}\right)(t), v\right)_{H}=\left(\partial_{t} y_{\alpha}(t), v\right)_{H} \\
\text { for all } v \in V \text { and a.a. } t \in(0, T) \\
y_{\alpha}(0)=w_{0, \alpha} \text { in } V, \quad \partial_{t} y_{\alpha}(0)=v_{0, \alpha}+u_{0, \alpha} \text { in } V^{\prime}, \quad u_{\alpha}(0)=u_{0, \alpha} \text { in } H .
\end{array}
$$

We obtain the formulation of problem $\left(\mathbf{P}_{\beta}\right)$ by setting $\alpha=0$ in problem $\left(\mathbf{P}_{\alpha, \beta}\right)$.

Problem $\left(\mathbf{P}_{\beta}\right)$. Find $(y, u, \xi)$ satisfying

$$
\begin{array}{r}
y \in W^{2,1}\left(0, T ; V^{\prime}\right) \cap W^{1, \infty}(0, T ; H) \cap L^{\infty}(0, T ; V) \\
u \in H^{1}\left(0, T ; V^{\prime}\right) \cap C^{0}([0, T] ; H) \cap L^{2}(0, T ; V) \\
\xi \in L^{2}(Q), \quad u \in D(\gamma) \text { and } \xi \in \gamma(u) \text { a.e. in } Q \\
\left\langle\partial_{t}^{2} y(t), v\right\rangle+\beta(\nabla y(t), \nabla v)_{H}=\beta(\nabla(1 * u)(t), \nabla v)_{H}+\langle f(t), v\rangle \\
\quad \text { for all } v \in V \text { and a.a. } t \in(0, T) \\
\left\langle\partial_{t} u(t), v\right\rangle+(\nabla u(t), \nabla v)_{H}+(\xi(t), v)_{H}+(g(u)(t), v)_{H}=\left(\partial_{t} y(t), v\right)_{H} \\
\quad \text { for all } v \in V \text { and a.a. } t \in(0, T) \\
y(0)=w_{0} \text { in } V, \quad \partial_{t} y(0)=v_{0}+u_{0} \text { in } V^{\prime}, \quad u(0)=u_{0} \text { in } H .
\end{array}
$$

Proposition 2.1 (Well-posedness). Under the assumptions (2.2) -(2.7) for both the families of data $\mathcal{D}_{\alpha}$ and $\mathcal{D}$, the two problems $\left(\boldsymbol{P}_{\alpha, \beta}\right)$ and $\left(\boldsymbol{P}_{\beta}\right)$ admit unique solutions $\left(y_{\alpha}, u_{\alpha}, \xi_{\alpha}\right)$ and $(y, u, \xi)$, respectively.

For the well-posedness of problem $\left(\mathbf{P}_{\alpha, \beta}\right)$ we refer to [7, Theorem 2.1], while the proof of the corresponding result for $\left(\mathbf{P}_{\beta}\right)$ can be found in [9], with the warning that the reader should argue with $y-w_{0}$ (in place of $y$ ) since in the framework of [9] the initial value for the related variable is 0 .

In this work, we are interested in the limit as $\alpha \searrow 0$ for problem $\left(\mathbf{P}_{\alpha, \beta}\right)$; so, what really affect us are the estimates for $\left(y_{\alpha}, u_{\alpha}, \xi_{\alpha}\right)$ independent of $\alpha$, rather then mere regularity results for problem $\left(\mathbf{P}_{\beta}\right)$. For this reason, all the results are stated in terms of the two families of data $\mathcal{D}_{\alpha}$ and $\mathcal{D}$.

Theorem 2.2 (Convergence as $\alpha \searrow 0$ ). Assume (2.2) $-(2.8)$ and let the families of data $\mathcal{D}_{\alpha}$ and $\mathcal{D}$ satisfy

$$
\begin{gathered}
f_{\alpha} \rightarrow f \quad \text { in } W^{1,1}\left(0, T ; V^{\prime}\right)+L^{1}(0, T ; H) \\
w_{0, \alpha} \rightarrow w_{0} \quad \text { in } V, \quad v_{0, \alpha} \rightarrow v_{0}, u_{0, \alpha} \rightarrow u_{0} \quad \text { in } H .
\end{gathered}
$$

Then, the convergences

$$
\begin{gathered}
y_{\alpha} \rightarrow^{*} y \quad \text { in } W^{1, \infty}(0, T ; H) \cap L^{\infty}(0, T ; V) \\
u_{\alpha} \rightarrow u \quad \text { in } H^{1}\left(0, T ; V^{\prime}\right) \cap L^{2}(0, T ; V), \quad \xi_{\alpha} \rightarrow \xi \quad \text { in } L^{2}(Q)
\end{gathered}
$$

hold as $\alpha \searrow 0$. 
Now, we strenghten the hypotheses on the initial data and on $f$, in order to obtain a strong solution to problem $\left(\mathbf{P}_{\beta}\right)$. In addition, we are able to provide an estimate on the convergence error, when $\alpha \searrow 0$. Let us suppose that

$$
\begin{gathered}
f_{\alpha} \in W^{1,1}(0, T ; H)+L^{1}(0, T ; V) \\
w_{0, \alpha} \in W, \quad v_{0, \alpha} \in V, \quad u_{0, \alpha} \in V \\
\left\|f_{\alpha}\right\|_{W^{1,1}(0, T ; H)+L^{1}(0, T ; V)}+\left\|w_{0, \alpha}\right\|_{W}+\left\|v_{0, \alpha}\right\|_{V}+\left\|u_{0, \alpha}\right\|_{V} \leq c .
\end{gathered}
$$

Theorem 2.3 (Regularity and strong solution). Assume (2.2)-(2.8). In addition, let (2.21) - 2.25) hold for $\mathcal{D}_{\alpha}$ and $\mathcal{D}$. Then the solution $(y, u, \xi)$ of problem $\left(\boldsymbol{P}_{\beta}\right)$ fulfills

$$
\begin{gathered}
y \in W^{2,1}(0, T ; H) \cap W^{1, \infty}(0, T ; V) \cap L^{\infty}(0, T ; W) \\
u \in H^{1}(0, T ; H) \cap C^{0}([0, T] ; V) \cap L^{2}(0, T ; W) .
\end{gathered}
$$

In particular, $(y, u, \xi)$ solves a strong reformulation of problem $\left(\boldsymbol{P}_{\beta}\right)$, namely,

$$
\begin{gathered}
\partial_{t}^{2} y-\beta \Delta y=-\beta \Delta(1 * u)+f \quad \text { a.e. in } Q \\
\partial_{t} u-\Delta u+\xi+g(u)=\partial_{t} y, \quad \xi \in \gamma(u) \quad \text { a.e. in } Q \\
\partial_{n} y=\partial_{n} u=0 \quad \text { a.e. on } \Gamma \times[0, T] .
\end{gathered}
$$

Theorem 2.4 (First error estimate). Let the assumptions (2.2) $-(2.8)$ and (2.23) (2.25) hold; if the inequalities

$$
\begin{gathered}
\left\|f_{\alpha}-f\right\|_{W^{1,1}\left(0, T ; V^{\prime}\right)+L^{1}(0, T ; H)} \leq c \alpha^{1 / 2} \\
\left\|w_{0, \alpha}-w_{0}\right\|_{V}+\left\|v_{0, \alpha}-v_{0}\right\|_{H}+\left\|u_{0, \alpha}-u_{0}\right\|_{H} \leq c \alpha^{1 / 2}
\end{gathered}
$$

are fulfilled, then the following estimate

$$
\left\|y_{\alpha}-y\right\|_{W^{1, \infty}(0, T ; H) \cap L^{\infty}(0, T ; V)}+\left\|u_{\alpha}-u\right\|_{L^{\infty}(0, T ; H) \cap L^{2}(0, T ; V)} \leq c_{\beta} \alpha^{1 / 2}
$$

holds for the convergence error.

Remark 2.5. Thanks to the informations we get on $u_{\alpha}-u$, and being $y=w+1 * u$, the estimate for $y_{\alpha}-y$ entails an analogous one for $w_{\alpha}-w$, which was the original variable of the problem. The same will happen for all the estimates presented in the paper.

The subsequent result provides an $L^{\infty}$ estimate on $u$. For $s \in D(\gamma)$, let $\gamma^{0}(s)$ be the element of $\gamma(s)$ having minimum modulus; we ask that

$$
\begin{gathered}
u_{0, \alpha} \in W, \quad u_{0, \alpha} \in D(\gamma) \quad \text { a.e. in } \Omega, \quad \gamma^{0}\left(u_{0, \alpha}\right) \in H \\
\left\|u_{0, \alpha}\right\|_{W}+\left\|\gamma^{0}\left(u_{0, \alpha}\right)\right\|_{H} \leq c
\end{gathered}
$$


Theorem 2.6 (Regularity for $u$ ). Assume (2.2)-(2.8) and let $\mathcal{D}_{\alpha}$, D satisfy the conditions (2.21) -(2.25), (2.33) -(2.34). Then the solutions $\left(y_{\alpha}, u_{\alpha}, \xi_{\alpha}\right),(y, u, \xi)$ of the respective problems $\left(\boldsymbol{P}_{\alpha, \beta}\right),\left(\boldsymbol{P}_{\beta}\right)$ fulfill

$$
u_{\alpha}, u \in W^{1, \infty}(0, T ; H) \cap H^{1}(0, T ; V) \cap L^{\infty}(0, T ; W) .
$$

All the results stated above hold for a domain $\Omega \subseteq \mathbb{R}^{N}$, where the dimension $N$ is arbitrary. Note in particular that (2.35) implies $u_{\alpha}, u \in C^{0}\left([0, T] ; H^{s}(\Omega)\right)$ for all $s<2$ (cf., e.g., [23, Sect. 8, Cor. 4]). On the other hand, il we let $N \leq 3$, the Sobolev embedding $H^{s}(\Omega) \hookrightarrow C^{0}(\bar{\Omega})$, holding for $s>3 / 2$, ensures that

$$
u \in C^{0}(\bar{Q}) .
$$

The subsequent results deal with the particular case, which is still physically significative, in which $\gamma$ is a smooth function, defined on the whole of $\mathbb{R}$. We require that

$$
\begin{aligned}
\gamma: \mathbb{R} \longrightarrow \mathbb{R} \quad \text { is single-valued and locally Lipschitz-continuous } \\
f_{\alpha} \in L^{2}(Q), \quad u_{0, \alpha} \in H^{3}(\Omega) \cap W, \quad\left\|f_{\alpha}\right\|_{L^{2}(Q)}+\left\|u_{0, \alpha}\right\|_{H^{3}(\Omega)} \leq c .
\end{aligned}
$$

Let us point out that the conditions (2.36) and (2.37) together entail (2.33) $-(2.34)$, because $\gamma^{0}\left(u_{0, \alpha}\right)=\gamma\left(u_{0, \alpha}\right)$ remains bounded in $L^{\infty}(\Omega)(\hookrightarrow H)$.

Theorem 2.7 (Further regularity on $u$ ). Assume (2.2) $-(2.8)$ and let $\mathcal{D}_{\alpha}$ and $\mathcal{D}$ fulfill (2.21) -(2.25) and (2.37); we also ask that $\gamma$ satisfy (2.36). Then the solutions $\left(y_{\alpha}, u_{\alpha}, \xi_{\alpha}\right)$ and $(y, u, \xi)$ of the respective problems $\left(\boldsymbol{P}_{\alpha, \beta}\right)$ and $\left(\boldsymbol{P}_{\beta}\right)$ satisfy

$$
u_{\alpha}, u \in H^{2}(0, T ; H) \cap W^{1, \infty}(0, T ; V) \cap H^{1}(0, T ; W) .
$$

Finally, we provide regularity enough to obtain an $L^{\infty}$ bound on $\partial_{t} y$ and a better estimate on the convergence error. Let

$$
\begin{gathered}
f_{\alpha} \in W^{2,1}(0, T ; H)+W^{1,1}(0, T ; V) \\
v_{0, \alpha} \in W, \quad \alpha \Delta v_{0, \alpha}+\beta \Delta w_{0, \alpha}+f_{\alpha}(0) \in V \\
\left\|f_{\alpha}\right\|_{W^{2,1}(0, T ; H)+W^{1,1}(0, T ; V)}+\left\|v_{0, \alpha}\right\|_{W}+\left\|\alpha \Delta v_{0, \alpha}+\beta \Delta w_{0, \alpha}+f_{\alpha}(0)\right\|_{V} \leq c .
\end{gathered}
$$

Theorem 2.8 (Further regularity on y). Let (2.2) -(2.8), (2.21)-(2.25) and (2.36) (2.40) hold. Then the solutions $\left(y_{\alpha}, u_{\alpha}, \xi_{\alpha}\right)$ and $(y, u, \xi)$ of the respective problems $\left(\boldsymbol{P}_{\alpha, \beta}\right)$ and $\left(\boldsymbol{P}_{\beta}\right)$ satisfy

$$
y_{\alpha}, y \in W^{2, \infty}(0, T ; V) \cap W^{1, \infty}(0, T ; W) .
$$


Theorem 2.9 (Second error estimate). Assume (2.2) -(2.8), (2.23) $-(2.25)$, (2.36) (2.40) and let the inequalities

$$
\begin{gathered}
\left\|f_{\alpha}-f\right\|_{W^{1,1}\left(0, T ; V^{\prime}\right)+L^{1}(0, T ; H)} \leq c \alpha \\
\left\|w_{0, \alpha}-w_{0}\right\|_{V}+\left\|v_{0, \alpha}-v_{0}\right\|_{H}+\left\|u_{0, \alpha}-u_{0}\right\|_{V} \leq c \alpha
\end{gathered}
$$

be satisfied. Then, the following estimate

$$
\begin{aligned}
& \left\|y_{\alpha}-y\right\|_{W^{1, \infty}(0, T ; H) \cap L^{\infty}(0, T ; V)} \\
& \quad+\left\|u_{\alpha}-u\right\|_{H^{1}(0, T ; H) \cap C^{0}([0, T] ; V) \cap L^{2}(0, T ; W)} \leq c_{\beta} \alpha
\end{aligned}
$$

holds true for the convergence error. Moreover, if

$$
\left\|f_{\alpha}-f\right\|_{W^{1,1}(0, T ; H)+L^{1}(0, T ; V)}+\left\|w_{0, \alpha}-w_{0}\right\|_{W}+\left\|v_{0, \alpha}-v_{0}\right\|_{V} \leq c \alpha^{1 / 2},
$$

then we also have

$$
\left\|y_{\alpha}-y\right\|_{W^{1, \infty}(0, T ; V) \cap L^{\infty}(0, T ; W)} \leq c_{\beta} \alpha^{1 / 2} .
$$

Remark 2.10. Focusing on regularity of the domain $\Omega$, all the proofs contained in this paper work if standards results on Sobolev embedding and elliptic regularity apply. For instance, if $\Omega \subseteq \mathbb{R}^{3}$ is a convex polyhedron, then all the results of this paper remain true.

\section{$3 \quad$ Preliminary results and convergence as $\alpha \searrow 0$}

For the reader's convenience, let us recall some results widely exploited in this paper. If $a, b \in L^{2}(Q)$ and $\sigma>0$ is an arbitrary parameter, by the Hölder and Young inequalities it is easy to infer that

$$
\int_{Q_{t}} a b \leq \frac{1}{2 \sigma} \int_{0}^{t}\|a(s)\|_{H}^{2} d s+\frac{\sigma}{2} \int_{0}^{t}\|b(s)\|_{H}^{2} d s .
$$

Furthermore, if $a \in H^{1}(0, T ; H)$, by the fundamental theorem of calculus and the Hölder inequality we have

$$
\|a(t)\|_{H}^{2}=\left\|a(0)+\int_{0}^{t} \partial_{t} a(s) d s\right\|_{H}^{2} \leq 2\|a(0)\|_{H}^{2}+2 T \int_{0}^{t}\left\|\partial_{t} a(s)\right\|_{H}^{2} d s .
$$

Finally, the following lemma states a well-known property of parabolic problems. For the sake of completeness, let us report a short proof.

Lemma 3.1. Let $h \in L^{2}(Q)$ and $z_{0} \in V$. Then, the problem

$$
\begin{gathered}
\partial_{t} z-\Delta z=h \text { a.e. in } Q \\
\partial_{n} z=0 \quad \text { a.e. on } \Gamma \times[0, T], \quad z(0)=z_{0} \quad \text { a.e. in } \Omega
\end{gathered}
$$

admits exactly one solution, which fulfills

$$
\|z\|_{H^{1}(0, T ; H) \cap C^{0}([0, T] ; V) \cap L^{2}(0, T ; W)} \leq c\left\{\left\|z_{0}\right\|_{V}+\|h\|_{L^{2}(Q)}\right\} .
$$


Proof. The existence of a solution for this problem can be proved via an approximationpassage to the limit scheme, such as, for instance, the Faedo-Galerkin method. The estimates needed to pass to the limit, by compactness arguments, are now formally provided. We test the equation with $\partial_{t} z$ and integrate over $Q_{t}$, obtaining:

$$
\int_{0}^{t}\left\|\partial_{t} z(s)\right\|_{H}^{2} d s+\frac{1}{2}\|\nabla z(t)\|_{H}^{2} \leq \frac{1}{2}\left\|\nabla z_{0}\right\|_{H}^{2}+\frac{1}{2} \int_{0}^{t}\|h(s)\|_{H}^{2} d s+\frac{1}{2} \int_{0}^{t}\left\|\partial_{t} z(s)\right\|_{H}^{2} d s .
$$

Such an inequality, combined with (3.2), directly yields

$$
\|z\|_{H^{1}(0, T ; H) \cap L^{\infty}(0, T ; V)} \leq c\left\{\left\|z_{0}\right\|_{V}+\|h\|_{L^{2}(Q)}\right\} .
$$

Then, by comparison in the equation and on account of the homogeneous boundary conditions, we obtain the estimate on $\|z\|_{L^{2}(0, T ; W)}$ as well. Setting $f \equiv 0$ and $z_{0}=0$, the previous inequality imply $z \equiv 0$, thus entailing the uniqueness of solutions.

Now we are ready to face the proof of Theorem 2.2. Firstly, we establish estimates independent of $\alpha$ on the solution $\left(y_{\alpha}, u_{\alpha}, \xi_{\alpha}\right)$ of problem $\left(\mathbf{P}_{\alpha, \beta}\right)$; then, we pass to the limit for a suitable subsequence, with compactness arguments. As we already know (cf. [9]) that Problem $\left(\mathbf{P}_{\beta}\right)$ has a unique solution, we will obtain the convergence of the whole family $\left(y_{\alpha}, u_{\alpha}, \xi_{\alpha}\right)$.

First a priori estimate We aim to test (2.12) by $\partial_{t} y_{\alpha}$ and (2.13) by $u_{\alpha}$ : as the former could be only formal due to regularity (2.9), we adopt the technique of singular perturbations to regularize $y_{\alpha}$; all the results we need can be found, for example, in 9, Prop. 6.1 and 6.2 , p. 505, Prop. 6.3, p. 506]. Hence, for $\varepsilon>0$ and a.a. $t \in(0, T)$, we define $y_{\alpha, \varepsilon}(t) \in V$ as the solution of the elliptic problem

$$
y_{\alpha, \varepsilon}(t)+\varepsilon^{2} J y_{\alpha, \varepsilon}(t)=y_{\alpha}(t),
$$

where $J$ is the Riesz isomorphism $V \longrightarrow V^{\prime}$. Since

$$
y_{\alpha} \in W^{2,1}\left(0, T ; V^{\prime}\right) \cap W^{1, \infty}(0, T ; H) \cap H^{1}(0, T ; V),
$$

then it follows that $y_{\alpha, \varepsilon} \in W^{2,1}(0, T ; V)$ and moreover, as $\varepsilon \searrow 0$,

$$
\begin{gathered}
y_{\alpha, \varepsilon} \rightarrow^{*} y_{\alpha} \text { in } W^{1, \infty}(0, T ; H) \\
y_{\alpha, \varepsilon} \rightarrow y_{\alpha} \text { in } H^{1}(0, T ; V) \text {, whence } y_{\alpha, \varepsilon} \longrightarrow y_{\alpha} \text { in } C^{0}([0, T] ; V) .
\end{gathered}
$$

We begin with testing equation (2.13) with $u_{\alpha}(t) \in V$ and integrating over $Q_{t}$. Recalling that $\gamma$ is monotone and $g$ is Lipschitz-continuous, it is straightforward to get

$$
\begin{aligned}
& \frac{1}{2}\left\|u_{\alpha}(t)\right\|_{H}^{2}+\int_{0}^{t}\left\|\nabla u_{\alpha}(s)\right\|_{H}^{2} d s \\
& \leq \frac{1}{2}\left\|u_{0, \alpha}\right\|_{H}^{2}+\frac{1}{2} \int_{0}^{t}\left\|\partial_{t} y_{\alpha}(s)\right\|_{H}^{2} d s+c \int_{0}^{t}\left\|u_{\alpha}(s)\right\|_{H}^{2} d s+c .
\end{aligned}
$$


Now we test equation (2.12) with $\partial_{t} y_{\alpha, \varepsilon}(t)$, integrate over $Q_{t}$, and pass to the limit as $\varepsilon \searrow 0$; it is convenient to treat each term separately. Thus, by [9, Prop. 6.2] and (3.5), we have

$$
\begin{gathered}
\lim _{\varepsilon \searrow 0} \int_{0}^{t}\left\langle\partial_{t}^{2} y_{\alpha}(s), \partial_{t} y_{\alpha, \varepsilon}(s)\right\rangle d s=\frac{1}{2}\left\|\partial_{t} y_{\alpha}(t)\right\|_{H}^{2}-\frac{1}{2}\left\|v_{0, \alpha}+u_{0, \alpha}\right\|_{H}^{2}, \\
\lim _{\varepsilon \searrow 0} \alpha \int_{Q_{t}} \nabla \partial_{t} y_{\alpha} \cdot \nabla \partial_{t} y_{\alpha, \varepsilon}=\alpha \int_{0}^{t}\left\|\nabla \partial_{t} y_{\alpha}(s)\right\|_{H}^{2} d s
\end{gathered}
$$

and, since $(v, z) \mapsto(\nabla v, \nabla z)_{H}$ gives a bilinear, symmetric, continuous and weakly coercive form in $V \times V$, by [9, Prop. 6.3] we find out that

$$
\lim _{\varepsilon \searrow 0} \beta \int_{Q_{t}} \nabla y_{\alpha} \cdot \nabla \partial_{t} y_{\alpha, \varepsilon}=\frac{\beta}{2}\left\|\nabla y_{\alpha}(t)\right\|_{H}^{2}-\frac{\beta}{2}\left\|\nabla w_{0, \alpha}\right\|_{H}^{2}
$$

Before dealing with the terms on the right-hand side, let us sum up and see that

$$
\begin{aligned}
& \frac{1}{2}\left\|\partial_{t} y_{\alpha}(t)\right\|_{H}^{2}+\alpha \int_{0}^{t}\left\|\nabla \partial_{t} y_{\alpha}(s)\right\|_{H}^{2} d s+\frac{\beta}{2}\left\|y_{\alpha}(t)\right\|_{V}^{2} \\
& \leq \frac{1}{2}\left\|v_{0, \alpha}+u_{0, \alpha}\right\|_{H}^{2}+\frac{\beta}{2}\left\|\nabla w_{0, \alpha}\right\|_{H}^{2}+\frac{\beta}{2}\left\|y_{\alpha}(t)\right\|_{H}^{2} \\
& +\lim _{\varepsilon \searrow 0}\left\{\alpha \int_{Q_{t}} \nabla u_{\alpha} \cdot \nabla \partial_{t} y_{\alpha, \varepsilon}+\beta \int_{Q_{t}} \nabla\left(1 * u_{\alpha}\right) \cdot \nabla \partial_{t} y_{\alpha, \varepsilon}+\int_{0}^{t}\left\langle f_{\alpha}(s), \partial_{t} y_{\alpha, \varepsilon}(s)\right\rangle d s\right\},
\end{aligned}
$$

where we have added the same quantity (cf. (3.2))

$$
\frac{\beta}{2}\left\|y_{\alpha}(t)\right\|_{H}^{2}\left(\leq \beta\left\|w_{0, \alpha}\right\|_{H}^{2}+T \beta \int_{0}^{t}\left\|\partial_{t} y_{\alpha}(s)\right\|_{H}^{2} d s\right)
$$

to both sides. We now deal with the right-hand side of (3.7) and note that

$$
\lim _{\varepsilon \searrow 0} \alpha \int_{Q_{t}} \nabla u_{\alpha} \cdot \nabla \partial_{t} y_{\alpha, \varepsilon}=\alpha \int_{Q_{t}} \nabla u_{\alpha} \nabla \partial_{t} y_{\alpha} \leq \alpha \int_{0}^{t}\left\|\nabla u_{\alpha}(s)\right\|_{H}^{2} d s+\frac{\alpha}{4} \int_{0}^{t}\left\|\nabla \partial_{t} y_{\alpha}(s)\right\|_{H}^{2} d s
$$

The other term involving $u_{\alpha}$ is treated by time integration by parts: thanks to (3.2) and (3.1) we have that

$$
\begin{aligned}
\lim _{\varepsilon \searrow 0} \beta \int_{Q_{t}} \nabla\left(1 * u_{\alpha}\right) & \cdot \nabla \partial_{t} y_{\alpha, \varepsilon}=\beta \lim _{\varepsilon \searrow 0}\left\{\left(\nabla\left(1 * u_{\alpha}\right)(t), \nabla y_{\alpha, \varepsilon}(t)\right)_{H}-\int_{Q_{t}} \nabla u_{\alpha} \cdot \nabla y_{\alpha, \varepsilon}\right\} \\
& \leq 5 \beta T \int_{0}^{t}\left\|\nabla u_{\alpha}(s)\right\|_{H}^{2} d s+\frac{\beta}{8}\left\|\nabla y_{\alpha}(t)\right\|_{H}^{2}+\frac{\beta}{4 T} \int_{0}^{t}\left\|\nabla y_{\alpha}(s)\right\|_{H}^{2} d s
\end{aligned}
$$

Finally, we fix a decomposition

$$
f_{\alpha}=f_{\alpha}^{(1)}+f_{\alpha}^{(2)}, \quad \text { with } \quad\left\|f_{\alpha}^{(1)}\right\|_{W^{1,1}\left(0, T ; V^{\prime}\right)}+\left\|f_{\alpha}^{(2)}\right\|_{L^{1}(0, T ; H)} \leq c .
$$


We integrate by parts in time the term containing $f^{(1)}$ and exploit (2.14) and (3.4)-(3.5) to obtain

$$
\begin{aligned}
& \lim _{\varepsilon \searrow 0} \int_{0}^{t}\left\langle f_{\alpha}^{(1)}(s), \partial_{t} y_{\alpha, \varepsilon}(s)\right\rangle d s \\
& =\lim _{\varepsilon \searrow 0}\left\{\left\langle f_{\alpha}^{(1)}(t), y_{\alpha, \varepsilon}(t)\right\rangle-\left\langle f_{\alpha}^{(1)}(0), y_{\alpha, \varepsilon}(0)\right\rangle-\int_{0}^{t}\left\langle\partial_{t} f_{\alpha}^{(1)}(s), y_{\alpha, \varepsilon}(s)\right\rangle d s\right\} \\
& \leq \frac{2}{\beta}\left\|f_{\alpha}^{(1)}(t)\right\|_{V^{\prime}}^{2}+\frac{\beta}{8}\left\|y_{\alpha}(t)\right\|_{V}^{2}+\frac{1}{2}\left\|f_{\alpha}^{(1)}(0)\right\|_{V^{\prime}}^{2} \\
& \quad+\frac{1}{2}\left\|w_{0, \alpha}\right\|_{V}^{2}+\int_{0}^{t}\left\|\partial_{t} f_{\alpha}^{(1)}(s)\right\|_{V^{\prime}}\left\|y_{\alpha}(s)\right\|_{V} d s \\
& \leq c_{\beta}\left\|f_{\alpha}^{(1)}\right\|_{L^{\infty}\left(0, T ; V^{\prime}\right)}^{2}+\frac{\beta}{8}\left\|y_{\alpha}(t)\right\|_{V}^{2}+\frac{1}{2}\left\|w_{0, \alpha}\right\|_{V}^{2}+\int_{0}^{t}\left\|\partial_{t} f_{\alpha}^{(1)}(s)\right\|_{V^{\prime}}\left\|y_{\alpha}(t)\right\|_{V} d s .
\end{aligned}
$$

Recalling (3.4), we easily get

$$
\lim _{\varepsilon \searrow 0} \int_{0}^{t}\left\langle f_{\alpha}^{(2)}(s), \partial_{t} y_{\alpha, \varepsilon}(s)\right\rangle d s=\int_{Q_{t}} f_{\alpha}^{(2)} \partial_{t} y_{\alpha} \leq \int_{0}^{t}\left\|f_{\alpha}^{(2)}(s)\right\|_{H}\left\|\partial_{t} y_{\alpha}(s)\right\|_{H} d s .
$$

Now, we collect all the inequalities referring to (3.7). Owing to (2.1), (2.8) and (3.8), we infer that

$$
\begin{aligned}
& \frac{1}{2}\left\|\partial_{t} y_{\alpha}(t)\right\|_{H}^{2}+\frac{3 \alpha}{4} \int_{0}^{t}\left\|\nabla \partial_{t} y_{\alpha}(s)\right\|_{H}^{2} d s+\frac{\beta}{4}\left\|y_{\alpha}(t)\right\|_{V}^{2} \\
& \leq c_{\beta}+c \int_{0}^{t}\left\|\partial_{t} y_{\alpha}(s)\right\|_{H}^{2} d s+(1+5 \beta T) \int_{0}^{t}\left\|\nabla u_{\alpha}(s)\right\|_{H}^{2} d s+c \int_{0}^{t}\left\|\nabla y_{\alpha}(s)\right\|_{H}^{2} d s \\
& \quad+\int_{0}^{t}\left\|\partial_{t} f_{\alpha}^{(1)}(s)\right\|_{V^{\prime}}\left\|y_{\alpha}(t)\right\|_{V} d s+\int_{0}^{t}\left\|f_{\alpha}^{(2)}(s)\right\|_{H}\left\|\partial_{t} y_{\alpha}(s)\right\|_{H} d s
\end{aligned}
$$

Then, let us multiply (3.6) by $(2+5 \beta T)$ and add the resulting inequality to (3.10), obtaining

$$
\begin{aligned}
& \left\|u_{\alpha}(t)\right\|_{H}^{2}+\int_{0}^{t}\left\|\nabla u_{\alpha}(s)\right\|_{H}^{2} d s+\frac{1}{2}\left\|\partial_{t} y_{\alpha}(t)\right\|_{H}^{2}+\frac{\alpha}{2} \int_{0}^{t}\left\|\nabla \partial_{t} y_{\alpha}(s)\right\|_{H}^{2} d s+\frac{\beta}{4}\left\|y_{\alpha}(t)\right\|_{V}^{2} \\
& \leq c_{\beta}\left\{1+\int_{0}^{t}\left\|u_{\alpha}(s)\right\|_{H}^{2} d s+\int_{0}^{t}\left\|\partial_{t} y_{\alpha}(s)\right\|_{H}^{2} d s+\int_{0}^{t}\left\|\nabla y_{\alpha}(s)\right\|_{H}^{2} d s\right\} \\
& \quad+\int_{0}^{t}\left\|\partial_{t} f_{\alpha}^{(1)}(s)\right\|_{V^{\prime}}\left\|y_{\alpha}(t)\right\|_{V} d s+\int_{0}^{t}\left\|f_{\alpha}^{(2)}(s)\right\|_{H}\left\|\partial_{t} y_{\alpha}(s)\right\|_{H} d s
\end{aligned}
$$

at this point, a generalised version of the Gronwall lemma, which is stated in [2, Teorema 2.1 , p. 245], allows us to conclude that

$$
\left\|y_{\alpha}\right\|_{W^{1, \infty}(0, T ; H) \cap L^{\infty}(0, T ; V)}+\sqrt{\alpha}\left\|y_{\alpha}\right\|_{H^{1}(0, T ; V)}+\left\|u_{\alpha}\right\|_{L^{\infty}(0, T ; H) \cap L^{2}(0, T ; V)} \leq c_{\beta} .
$$

Remark 3.2. Estimate (3.11) can be easily modified in order to provide the continuous dependence on the data for problem $\left(\mathbf{P}_{\alpha, \beta}\right)$. Indeed, taking the difference between the equations associated with two sets of data $\mathcal{D}_{\alpha, 1}$ and $\mathcal{D}_{\alpha, 2}$, the resulting system is formally analougous to $\left(\mathbf{P}_{\alpha, \beta}\right)$ and can be treated similarly; the only difference is the term in $g\left(u_{\alpha}\right)$, which is replaced by $g\left(u_{\alpha, 1}\right)-g\left(u_{\alpha, 2}\right)$. We obtain an estimate where the constant in the right-hand side is independent of $\alpha$. 
Second a priori estimate. In order to prove the next estimate, we need to approximate the graph $\gamma$ with its Yosida regularization; therefore, for all $\varepsilon>0$ we define

$$
\gamma_{\varepsilon}:=\frac{1}{\varepsilon}\left\{I-(I+\varepsilon \gamma)^{-1}\right\} \quad \text { and } \quad \phi_{\varepsilon}(s):=\min _{\tau \in \mathbb{R}}\left\{\frac{1}{2 \varepsilon}|\tau-s|^{2}+\phi(\tau)\right\} \quad \text { for } s \in \mathbb{R},
$$

where $I$ denotes the identity on $\mathbb{R}$. We remind that $\phi_{\varepsilon}$ is a nonnegative and differentiable function, $\gamma_{\varepsilon}$ is increasing and Lipschitz-continuous: moreover, we have that

$$
\phi_{\varepsilon}^{\prime}(s)=\gamma_{\varepsilon}(s), \quad 0 \leq \gamma_{\varepsilon}^{\prime}(s) \leq \frac{1}{\varepsilon}, \quad 0 \leq \phi_{\varepsilon}(s) \leq \phi(s) \quad \text { for all } s \in \mathbb{R} \text { and } \varepsilon>0
$$

(see, e.g., [4, Prop. 2.6, p. 28 and Prop. 2.11, p. 39] or [3, pp. 57-58]). We establish an estimate for the solution $\left(y_{\alpha}, u_{\alpha}, \xi_{\alpha}\right)$ to problem $\left(\mathbf{P}_{\alpha, \beta}\right)$, in which we have replaced $\gamma$ by $\gamma_{\varepsilon}$; then, the same estimate will hold for the original problem, because of the passage to the limit as $\varepsilon \searrow 0$ already detailed in [7].

We notice that $\gamma_{\varepsilon}(u)(t) \in V$ for a.a. $t$, due to the Lipschitz-continuity of $\gamma_{\varepsilon}$, and so it is an admissible test function for equation (2.13). The Lipschitz-continuity of $g$ and the formula

$$
\gamma_{\varepsilon}\left(u_{\alpha}\right) \partial_{t} u_{\alpha}=\frac{\partial}{\partial t} \phi_{\varepsilon}\left(u_{\alpha}\right)
$$

allow us to write

$$
\begin{aligned}
& \left\|\phi_{\varepsilon}\left(u_{\alpha}\right)(t)\right\|_{L^{1}(\Omega)}+\int_{0}^{t} \gamma_{\varepsilon}^{\prime}\left(u_{\alpha}\right)\left|\nabla u_{\alpha}\right|^{2}+\int_{0}^{t}\left\|\gamma_{\varepsilon}\left(u_{\alpha}\right)(s)\right\|_{H}^{2} d s \\
& \leq\left\|\phi_{\varepsilon}\left(u_{0, \alpha}\right)\right\|_{L^{1}(\Omega)}+\int_{0}^{t}\left\|\partial_{t} y_{\alpha}(s)\right\|_{H}^{2} d s+c \int_{0}^{t}\left\|u_{\alpha}(s)\right\|_{H}^{2} d s+c+\frac{1}{2} \int_{0}^{t}\left\|\gamma_{\varepsilon}\left(u_{\alpha}\right)(s)\right\|_{H}^{2} d s .
\end{aligned}
$$

We ignore the second term in the left-hand side, which is positive because of $\gamma_{\varepsilon}^{\prime} \geq 0$, and use (3.12), (2.8) and (3.11) to get

$$
\left\|\phi_{\varepsilon}\left(u_{\alpha}\right)(t)\right\|_{L^{1}(\Omega)}+\frac{1}{2} \int_{0}^{t}\left\|\gamma_{\varepsilon}\left(u_{\alpha}\right)(s)\right\|_{H}^{2} d s \leq c_{\beta} .
$$

As $\phi_{\varepsilon} \longrightarrow \phi$ pointwise (see [4, Prop. 2.11, p. 39]), by applying the Fatou lemma to the first term and passing to the limit as $\varepsilon \searrow 0$ we obtain

$$
\left\|\phi\left(u_{\alpha}\right)\right\|_{L^{\infty}\left(0, T ; L^{1}(\Omega)\right)}+\left\|\xi_{\alpha}\right\|_{L^{2}(Q)} \leq c_{\beta} .
$$

By comparison in the equation (2.13), we also deduce that

$$
\left\|\partial u_{\alpha}\right\|_{L^{2}\left(0, T ; V^{\prime}\right)} \leq c_{\beta} .
$$

Passage to the limit as $\alpha \searrow 0$. According to the estimates (3.11), (3.13) and (3.14), it is fair to assume, up to subsequences,

$$
\begin{gathered}
y_{\alpha} \rightarrow^{*} y \quad \text { in } W^{1, \infty}(0, T ; H) \cap L^{\infty}(0, T ; V) \\
u_{\alpha} \rightarrow u \quad \text { in } H^{1}\left(0, T ; V^{\prime}\right) \cap L^{2}(0, T ; V), \quad \xi_{\alpha} \rightarrow \xi \quad \text { in } L^{2}(Q) .
\end{gathered}
$$


The generalised Ascoli theorem and the Aubin-Lions lemma (see, e.g., [23, Sect. 8, Cor. 4]) thus imply

$$
\begin{gathered}
y_{\alpha} \longrightarrow y \quad \text { strongly in } C^{0}([0, T] ; H) \\
u_{\alpha} \longrightarrow u \text { strongly in } C^{0}\left([0, T] ; V^{\prime}\right) \text { and in } L^{2}(Q),
\end{gathered}
$$

and the Lipschitz-continuity of $g$ yields

$$
g\left(u_{\alpha}\right) \longrightarrow g(u) \text { strongly in } L^{2}(Q)
$$

as $\alpha \searrow 0$. With all this information and in view of (2.21), (2.22) it is not difficult to check that the limits $y, u, \xi$ form a triplet solving problem $\left(\mathbf{P}_{\beta}\right)$. The proof is analogous to the one developed in [7, Section 5]: in particular, let us point out that

$$
\lim _{\alpha \searrow 0} \int_{Q} \xi_{\alpha} u_{\alpha}=\int_{Q} \xi u
$$

which entails (see, e.g., [3, Prop. 1.1, p. 42]) (2.17). Moreover, the regularity $W^{2,1}\left(0, T ; V^{\prime}\right)$ for $y$ can be recovered a posteriori by comparing terms of (2.18).

\section{Strong solutions and first error estimate}

This section is devoted to the proofs of Theorems 2.3 and 2.4. Regularity results for problem $\left(\mathbf{P}_{\beta}\right)$ are obtained by establishing estimates for $\left(y_{\alpha}, u_{\alpha}, \xi_{\alpha}\right)$ independent of $\alpha$; then Theorem 2.2 and the weak or weak star compactness yield the desired regularity for $(y, u, \xi)$. About the end of the section, we also discuss briefly how the thesis of Theorem 2.6 follows from the furher requirements in (2.33)-(2.34).

We assume that $\mathcal{D}_{\alpha}$ and $\mathcal{D}$ satisfy hypotheses (2.23) -(2.25). Under these assumptions, from [7, Theorem 2.2] we know that

$$
\begin{gathered}
y_{\alpha} \in W^{2,1}(0, T ; H) \cap W^{1, \infty}(0, T ; V) \cap H^{1}(0, T ; W) \\
u_{\alpha} \in H^{1}(0, T ; H) \cap C^{0}([0, T] ; V) \cap L^{2}(0, T ; W)
\end{gathered}
$$

and $\left(y_{\alpha}, u_{\alpha}, \xi_{\alpha}\right)$ solves Problem $\left(\mathbf{P}_{\alpha, \beta}\right)$ in a strong sense, that is, $y_{\alpha}$ and $u_{\alpha}$ satisfy

$$
\begin{gathered}
\partial_{t}^{2} y_{\alpha}-\alpha \Delta \partial_{t} y_{\alpha}-\beta \Delta y_{\alpha}=-\alpha \Delta u_{\alpha}-\beta \Delta\left(1 * u_{\alpha}\right)+f_{\alpha} \quad \text { a.e. in } Q \\
\partial_{t} u_{\alpha}-\Delta u_{\alpha}+\xi_{\alpha}+g\left(u_{\alpha}\right)=\partial_{t} y_{\alpha}, \quad \xi_{\alpha} \in \gamma\left(u_{\alpha}\right) \quad \text { a.e. in } Q \\
\partial_{n} y_{\alpha}=\partial_{n} u_{\alpha}=0 \quad \text { a.e. on } \Gamma \times(0, T)
\end{gathered}
$$

along with (2.14) , for every $\alpha \in(0,1)$.

Third a priori estimate. Thanks to (4.4)-(4.5), we can apply Lemma 3.1 with $z=u_{\alpha}, z_{0}=u_{0, \alpha}$, and $h=\partial_{t} y_{\alpha}-\xi_{\alpha}-g\left(u_{\alpha}\right)$. Indeed, in view of the condition in (2.25) for the initial datum, and owing to (2.5) and to estimates (3.11) and (3.13), from (3.3) it follows that

$$
\left\|u_{\alpha}\right\|_{H^{1}(0, T ; H) \cap L^{\infty}(0, T ; V) \cap L^{2}(0, T ; W)} \leq c_{\beta} .
$$


Fourth a priori estimate. By virtue of (4.2), we can plainly multiply equation (4.4) by $-\Delta \partial_{t} y_{\alpha}$ and integrate over $Q_{t}$. Thus, we obtain

$$
\begin{gathered}
\frac{1}{2}\left\|\nabla \partial_{t} y_{\alpha}(t)\right\|_{H}^{2}+\alpha \int_{0}^{t}\left\|\Delta \partial_{t} y_{\alpha}(s)\right\|_{H}^{2} d s+\frac{\beta}{2}\left\|\Delta y_{\alpha}(t)\right\|_{H}^{2} \leq \frac{1}{2}\left\|\nabla\left(v_{0, \alpha}+u_{0, \alpha}\right)\right\|_{H}^{2} \\
+\frac{\beta}{2}\left\|\Delta w_{0, \alpha}\right\|_{H}^{2}+\alpha \int_{Q_{t}} \Delta u_{\alpha} \Delta \partial_{t} y_{\alpha}+\beta \int_{Q_{t}} \Delta\left(1 * u_{\alpha}\right) \Delta \partial_{t} y_{\alpha}-\int_{Q_{t}} f_{\alpha} \Delta \partial_{t} y_{\alpha} .
\end{gathered}
$$

A simple application of the Hölder inequality allow us to infer that

$$
\alpha \int_{Q_{t}} \Delta u_{\alpha} \Delta \partial_{t} y_{\alpha} \leq \frac{\alpha}{2} \int_{0}^{t}\left\|\Delta u_{\alpha}(s)\right\|_{H}^{2} d s+\frac{\alpha}{2} \int_{0}^{t}\left\|\Delta \partial_{t} y_{\alpha}(s)\right\|_{H}^{2} d s .
$$

We deal with the subsequent term integrating by parts in time:

$$
\begin{gathered}
\beta \int_{Q_{t}} \Delta\left(1 * u_{\alpha}\right) \Delta \partial_{t} y_{\alpha} \leq 2 \beta\left\|1 * \Delta u_{\alpha}(t)\right\|_{H}^{2}+\frac{\beta}{8}\left\|\Delta y_{\alpha}(t)\right\|_{H}^{2}-\beta \int_{Q_{t}} \Delta u_{\alpha} \Delta y_{\alpha} \\
\leq \beta(4 T+1) \int_{0}^{t}\left\|\Delta u_{\alpha}(s)\right\|_{H}^{2} d s+\frac{\beta}{8}\left\|\Delta y_{\alpha}(t)\right\|_{H}^{2}+\frac{\beta}{4} \int_{0}^{t}\left\|\Delta y_{\alpha}(s)\right\|_{H}^{2} d s .
\end{gathered}
$$

Now, we choose a split

$$
f_{\alpha}=f_{\alpha}^{(1)}+f_{\alpha}^{(2)}, \quad \text { with } \quad\left\|f_{\alpha}^{(1)}\right\|_{W^{1,1}(0, T ; H)}+\left\|f_{\alpha}^{(2)}\right\|_{L^{1}(0, T ; V)} \leq c
$$

in order to estimate the term with $f_{\alpha}$. Concerning $f_{\alpha}^{(1)}$, we integrate by parts in time and recall (2.25):

$$
\begin{gathered}
-\int_{Q_{t}} f_{\alpha}^{(1)} \Delta \partial_{t} y_{\alpha}=-\left(f_{\alpha}^{(1)}(t), \Delta y_{\alpha}(t)\right)_{H}+\left(f_{\alpha}^{(1)}(0), \Delta w_{0, \alpha}\right)_{H}+\int_{Q_{t}} \partial_{t} f_{\alpha}^{(1)} \Delta y_{\alpha} \\
\leq \frac{2}{\beta}\left\|f_{\alpha}^{(1)}(t)\right\|_{H}^{2}+\frac{\beta}{8}\left\|\Delta y_{\alpha}(t)\right\|_{H}^{2}+\int_{0}^{t}\left\|\partial_{t} f_{\alpha}^{(1)}(s)\right\|_{H}\left\|\Delta y_{\alpha}(s)\right\|_{H} d s+c .
\end{gathered}
$$

An integration by parts in space leads to

$$
-\int_{Q t} f_{\alpha}^{(2)} \Delta \partial_{t} y_{\alpha} \leq \int_{0}^{t}\left\|\nabla f_{\alpha}^{(2)}(s)\right\|_{H}\left\|\nabla \partial_{t} y_{\alpha}(s)\right\|_{H} d s .
$$

Collecting all these inequalities and taking advantage of (2.25), (2.1), (4.6) and (4.8), from (4.7) we derive

$$
\begin{array}{r}
\frac{1}{2}\left\|\nabla \partial_{t} y_{\alpha}(t)\right\|_{H}^{2}+\frac{\alpha}{2} \int_{0}^{t}\left\|\Delta \partial_{t} y_{\alpha}(s)\right\|_{H}^{2} d s+\frac{\beta}{4}\left\|\Delta y_{\alpha}(t)\right\|_{H}^{2} \leq c_{\beta}+c \int_{0}^{t}\left\|\Delta y_{\alpha}(s)\right\|_{H}^{2} d s \\
+\int_{0}^{t}\left\|\partial_{t} f_{\alpha}^{(1)}(s)\right\|_{H}\left\|\Delta y_{\alpha}(s)\right\|_{H} d s+\int_{0}^{t}\left\|\nabla f_{\alpha}^{(2)}(s)\right\|_{H}\left\|\nabla \partial_{t} y_{\alpha}(s)\right\|_{H} d s .
\end{array}
$$

Hence, by means of the generalised Gronwall lemma (see, e.g., [2]) and regularity properties for elliptic problems, we obtain

$$
\left\|y_{\alpha}\right\|_{W^{1, \infty}(0, T ; V) \cap L^{\infty}(0, T ; W)}+\sqrt{\alpha}\left\|y_{\alpha}\right\|_{H^{1}(0, T ; W)} \leq c_{\beta} .
$$


Next, a comparison in equation (4.4) gives

$$
\left\|\partial_{t}^{2} y_{\alpha}-f_{\alpha}^{(2)}\right\|_{L^{2}(0, T ; H)} \leq c_{\beta} .
$$

Thus, we have collected all the estimates needed to prove Theorem 2.3. In particular, note that the regularity (2.26)-(2.27) is enssured for the limit functions $y$ and $u$, which actually satisfy (2.28)-(2.30).

Error equations. We have already shown the convergence as $\alpha \searrow 0$ for the solutions to problem $\left(\mathbf{P}_{\alpha, \beta}\right)$; as we want to study the speed of convergence, we start writing explicitely the equations for the error. We set

$$
\begin{gathered}
\widehat{y}_{\alpha}=y_{\alpha}-y, \quad \widehat{u}_{\alpha}=u_{\alpha}-u, \quad \widehat{\xi}_{\alpha}=\xi_{\alpha}-\xi \\
\widehat{f}_{\alpha}=f_{\alpha}-f, \quad \widehat{w}_{0, \alpha}=w_{0, \alpha}-w_{0}, \quad \widehat{v}_{0, \alpha}=v_{0, \alpha}-v_{0}, \quad \widehat{u}_{0, \alpha}=u_{0, \alpha}-u_{0}
\end{gathered}
$$

and subtract side by side the equations of the problems $\left(\mathbf{P}_{\alpha, \beta}\right)$ and $\left(\mathbf{P}_{\beta}\right)$, in their strong formulations:

$$
\begin{gathered}
\partial_{t}^{2} \widehat{y}_{\alpha}-\beta \Delta \widehat{y}_{\alpha}=\alpha \Delta \partial_{t} y_{\alpha}-\alpha \Delta u_{\alpha}-\beta \Delta\left(1 * \widehat{u}_{\alpha}\right)+\widehat{f}_{\alpha} \quad \text { a.e. in } Q \\
\partial_{t} \widehat{u}_{\alpha}-\Delta \widehat{u}_{\alpha}+\widehat{\xi}_{\alpha}+g\left(u_{\alpha}\right)-g(u)=\partial_{t} \widehat{y}_{\alpha} \quad \text { a.e. in } Q \\
\partial_{n} \widehat{y}_{\alpha}=0, \quad \partial_{n} \widehat{u}_{\alpha}=0 \quad \text { a.e. on } \Gamma \times(0, T) \\
\widehat{y}_{\alpha}(0)=\widehat{w}_{0, \alpha}, \quad \partial_{t} \widehat{y}_{\alpha}(0)=\widehat{v}_{0, \alpha}+\widehat{u}_{0, \alpha}, \quad \widehat{u}_{\alpha}(0)=\widehat{u}_{0, \alpha} \quad \text { a.e. in } \Omega .
\end{gathered}
$$

When necessary, we also split $\widehat{f}_{\alpha}=\widehat{f}_{\alpha}^{(1)}+\widehat{f}_{\alpha}^{(2)}$, where $f_{\alpha}^{(1)}, f_{\alpha}^{(2)}$ (and also $\left.f^{(1)}, f^{(2)}\right)$ fulfill (4.8), $\widehat{f}_{\alpha}^{(i)}=f_{\alpha}^{(i)}-f^{(i)}$ for $i=1,2$, and

$$
\left\|\widehat{f}_{\alpha}^{(1)}\right\|_{W^{1,1}\left(0, T ; V^{\prime}\right)}+\left\|\widehat{f}_{\alpha}^{(2)}\right\|_{L^{1}(0, T ; H)} \leq c \alpha^{1 / 2}
$$

First error estimate. Now we want to show Theorem 2.4. We multiply equation (4.13) by $\widehat{u}_{\alpha}$ and integrate over $Q_{t}$; on account of the monotonicity of $\gamma$ and the Lipschitzcontinuity of $g$, it is straightforward to get

$$
\begin{aligned}
& \frac{1}{2}\left\|\widehat{u}_{\alpha}(t)\right\|_{H}^{2}+\int_{0}^{t}\left\|\nabla \widehat{u}_{\alpha}(s)\right\|_{H}^{2} d s \\
& \leq \frac{1}{2}\left\|\widehat{u}_{0, \alpha}\right\|_{H}^{2}+c \int_{0}^{t}\left\|\widehat{u}_{\alpha}(s)\right\|_{H}^{2} d s+\frac{1}{2} \int_{0}^{t}\left\|\partial_{t} \widehat{y}_{\alpha}(s)\right\|_{H}^{2} d s .
\end{aligned}
$$

Next, we add $\beta \widehat{y}_{\alpha}$ to both sides of (4.12), then test with $\partial_{t} \widehat{y}_{\alpha}$ and integrate over $Q_{t}$. With the help of (4.15) and (3.2) we easily obtain

$$
\begin{aligned}
\frac{1}{2}\left\|\partial_{t} \widehat{y}_{\alpha}(t)\right\|_{H}^{2}+\frac{\beta}{2}\left\|\widehat{y}_{\alpha}(s)\right\|_{V}^{2} \leq \frac{1}{2}\left\|\widehat{v}_{0, \alpha}+\widehat{u}_{0, \alpha}\right\|_{H}^{2}+\frac{\beta}{2}\left\|\widehat{w}_{0, \alpha}\right\|_{V}^{2} \\
+\beta\left\|\widehat{w}_{0, \alpha}\right\|_{H}^{2}+T \beta \int_{0}^{t}\left\|\partial_{t} \widehat{y}_{\alpha}(s)\right\|_{H}^{2} d s+\alpha \int_{Q_{t}}\left(\Delta \partial_{t} y_{\alpha}-\Delta u_{\alpha}\right) \partial_{t} \widehat{y}_{\alpha} \\
\quad-\beta \int_{Q_{t}} \Delta\left(1 * \widehat{u}_{\alpha}\right) \partial_{t} \widehat{y}_{\alpha}+\int_{0}^{t}\left\langle\widehat{f}_{\alpha}^{(1)}(s), \partial_{t} \widehat{y}_{\alpha}(s)\right\rangle d s+\int_{Q_{t}} \widehat{f}_{\alpha}^{(2)} \partial_{t} \widehat{y}_{\alpha} .
\end{aligned}
$$


The term involving $\alpha$ is easily treated by the Hölder inequality and estimates (4.6), (4.10):

$$
\begin{aligned}
\alpha \int_{Q_{t}}\left(\Delta \partial_{t} y_{\alpha}-\Delta u_{\alpha}\right) \partial_{t} \widehat{y}_{\alpha} \leq & \frac{\alpha}{2} \int_{0}^{t} \alpha\left\|\Delta \partial_{t} y_{\alpha}(s)\right\|_{H}^{2} d s+\frac{\alpha^{2}}{2} \int_{0}^{t}\left\|\Delta u_{\alpha}(s)\right\|_{H}^{2} d s \\
& +\int_{0}^{t}\left\|\partial_{t} \widehat{y}_{\alpha}(s)\right\|_{H}^{2} d s \leq c_{\beta} \alpha+\int_{0}^{t}\left\|\partial_{t} \widehat{y}_{\alpha}(s)\right\|_{H}^{2} d s .
\end{aligned}
$$

Here, (2.1) has been used in the control of $\alpha^{2}$ by $\alpha$. For the subsequent term, we integrate by parts in space and time and recall (3.2):

$$
\begin{aligned}
& -\beta \int_{Q_{t}} \Delta\left(1 * \widehat{u}_{\alpha}\right) \partial_{t} \widehat{y}_{\alpha} \leq \beta\left(\nabla\left(1 * \widehat{u}_{\alpha}\right)(t), \nabla \widehat{y}_{\alpha}(t)\right)-\beta \int_{Q_{t}} \nabla \widehat{u}_{\alpha} \cdot \nabla \widehat{y}_{\alpha} \\
& \quad \leq 5 \beta T \int_{0}^{t}\left\|\nabla \widehat{u}_{\alpha}(s)\right\|_{H}^{2} d s+\frac{\beta}{8}\left\|\nabla \widehat{y}_{\alpha}(t)\right\|_{H}^{2}+\frac{\beta}{4 T} \int_{0}^{t}\left\|\nabla \widehat{y}_{\alpha}(s)\right\|_{H}^{2} d s .
\end{aligned}
$$

The next term in (4.18) is treated similarly as in (3.9). We infer that

$$
\begin{gathered}
\int_{0}^{t}\left\langle\widehat{f}_{\alpha}^{(1)}(s), \partial_{t} \widehat{y}_{\alpha}(s)\right\rangle d s \leq c_{\beta}\left\|\widehat{f}_{\alpha}^{(1)}\right\|_{L^{\infty}\left(0, T ; V^{\prime}\right)}^{2}+\frac{\beta}{8}\left\|\nabla \widehat{y}_{\alpha}(t)\right\|_{H}^{2}+\frac{\beta}{4}\left\|\widehat{w}_{0, \alpha}\right\|_{H}^{2} \\
\quad+\frac{\beta T}{4} \int_{0}^{t}\left\|\partial_{t} \widehat{y}_{\alpha}(s)\right\|_{H}^{2} d s+\frac{1}{2}\left\|\widehat{w}_{0, \alpha}\right\|_{V}^{2}+\int_{0}^{t}\left\|\partial_{t} \widehat{f}_{\alpha}^{(1)}(s)\right\|_{V^{\prime}}\left\|\widehat{y}_{\alpha}(s)\right\|_{V} d s
\end{gathered}
$$

while the other contribution of the source term is easily estimated by the Hölder inequality:

$$
\int_{Q t} \widehat{f}_{\alpha}^{(2)} \partial_{t} \widehat{y}_{\alpha} \leq \int_{0}^{t}\left\|\widehat{f}_{\alpha}^{(2)}(s)\right\|_{H}\left\|\partial_{t} \widehat{y}_{\alpha}(s)\right\|_{H} d s
$$

Now we multiply (4.17) by $(1+5 \beta T)$ and add the resulting inequality to (4.18), side by side. In view of (4.19) $-(4.22)$ and thanks to (2.31) $-(2.32)$, we deduce that

$$
\begin{aligned}
& \frac{1}{2}\left\|\widehat{u}_{\alpha}(t)\right\|_{H}^{2}+\int_{0}^{t}\left\|\nabla \widehat{u}_{\alpha}(s)\right\|_{H}^{2} d s+\frac{1}{2}\left\|\partial_{t} \widehat{y}_{\alpha}(t)\right\|_{H}^{2}+\frac{\beta}{4}\left\|\widehat{y}_{\alpha}(t)\right\|_{V}^{2} \\
& \leq c_{\beta} \alpha+c \int_{0}^{t}\left\|\widehat{u}_{\alpha}(s)\right\|_{H}^{2} d s+c \int_{0}^{t}\left\|\partial_{t} \widehat{y}_{\alpha}(s)\right\|_{H}^{2} d s+c \int_{0}^{t}\left\|\nabla \widehat{y}_{\alpha}(s)\right\|_{H}^{2} d s \\
& \quad+\int_{0}^{t}\left\|\partial_{t} \widehat{f}_{\alpha}^{(1)}(s)\right\|_{V^{\prime}}\left\|\widehat{y}_{\alpha}(s)\right\|_{V} d s+\int_{0}^{t}\left\|\widehat{f}_{\alpha}^{(2)}(s)\right\|_{H}\left\|\partial_{t} \widehat{y}_{\alpha}(s)\right\|_{H} d s
\end{aligned}
$$

hence, the generalised Gronwall lemma (see, e.g., [2]) and (2.31) again entail the thesis of Theorem 2.4.

A regularity result on $u$. Let us formally describe how Theorem 2.6 can be proved: you differentiate the equation in (4.4) with respect to time and then test by $\partial_{t} y_{\alpha}$, integrating over $Q_{t}$ with the help of the further initial condition for $\partial_{t} y_{\alpha}(0)$. Such a condition can be still inferred from (4.4) and, thanks to (2.34), the initial values $\partial_{t} y_{\alpha}(0)$ turn out to be bounded in $H$. Moreover, you exploit the facts that

$$
\int_{Q_{t}} \partial_{t} \xi_{\alpha} \partial_{t} y_{\alpha} \geq 0
$$


and that $\partial_{t}^{2} y_{\alpha}$ stays uniformly bounded in $L^{1}(0, T ; H)$ (cf. (4.11) and (2.23)).

However, for a rigorous proof we refer to [7, Section 7], where the sketched estimate is carried out properly, in the setting provided by the Faedo-Galerkin approximation and the Yosida regularisation of the graph $\gamma$. Since the estimate is independent of $\alpha$, for the proof, as a by-product we have the desired inequality

$$
\left\|u_{\alpha}\right\|_{W^{1, \infty}(0, T ; H) \cap H^{1}(0, T ; V) \cap L^{\infty}(0, T ; W)} \leq c_{\beta},
$$

which pass to the limit as $\alpha \searrow 0$ via compactness argument.

\section{The case of a smooth potential}

In what follows, we assume that $\gamma$ is a single-valued, non decreasing, locally Lipschitzcontinuous function, defined on the whole of $\mathbb{R}$ (see (2.3) and (2.36) ). We have already pointed out that this assumption is restrictive but not unrealistic, as the example of the 'double-well' potential (1.19) shows.

Since the hypotheses we consider on the data are strong enough to guarantee (4.24), and consequently the estimate

$$
\left\|u_{\alpha}\right\|_{L^{\infty}(Q)} \leq c_{\beta},
$$

we have that $\xi_{\alpha}=\gamma\left(u_{\alpha}\right)$ is bounded independently of $\alpha$ in $L^{\infty}(Q)$. Furthermore, by the local Lipschitz-continuity of $\gamma$, without loss of generality we can set $\xi_{\alpha}=0$ in (2.13) (and (4.4) $), \xi=0$ in (2.19) (and (2.29) $), \widehat{\xi}_{\alpha}=0$ in (4.13) by modifying $g$ in order to take account of $\gamma$ too.

Further regularity on $u$. As we are interested in showing Theorem 2.7, we consider equation (2.13) and derive it, with respect to time. We get a first-order equation for a new unknown $z_{\alpha}=\partial_{t} u_{\alpha}$, which is complemented by Neumann homogeneus boundary conditions and the suitable initial condition, obtained by setting $t=0$ in (4.4):

$$
\begin{gathered}
\partial_{t} z_{\alpha}-\Delta z_{\alpha}=-\partial_{t}\left(g\left(u_{\alpha}\right)\right)+\partial_{t}^{2} y_{\alpha} \quad \text { a.e. in } Q, \\
\partial_{n} z_{\alpha}=0 \quad \text { a.e. in } \Gamma \times(0, T) \\
z_{\alpha}(0)=\Delta u_{0, \alpha}-g\left(u_{0, \alpha}\right)+v_{0, \alpha}+u_{0, \alpha} \quad \text { a.e. in } \Omega .
\end{gathered}
$$

We notice that the terms $\partial_{t}\left(g\left(u_{\alpha}\right)\right)$ and $\partial_{t}^{2} y_{\alpha}$ are bounded in $L^{2}(Q)$ due to (2.5), (4.6) and (4.11), (2.37). Moreover, (2.25) and (2.37) imply that

$$
\Delta u_{0, \alpha}-g\left(u_{0, \alpha}\right)+v_{0, \alpha}+u_{0, \alpha} \text { is bounded in } V
$$

independently of $\alpha$. Thus, we can apply Lemma 3.1 and, on account of (4.24), the inequality (3.3) leads the estimate

$$
\left\|u_{\alpha}\right\|_{H^{2}(0, T ; H) \cap W^{1, \infty}(0, T ; V) \cap H^{1}(0, T ; W)} \leq c_{\beta},
$$

whence the thesis of Theorem 2.7 follows. 
Further regularity on $\partial_{t} y$. Here, we want to prove Theorem 2.8, whose assumptions are now supposed to be in force. Recalling (4.3), (4.5) and (2.14), it turns out that $v_{\alpha}=\partial_{t} y_{\alpha}$ formally satisfies

$$
\begin{gathered}
\partial_{t}^{2} v_{\alpha}-\alpha \Delta \partial_{t} v_{\alpha}-\beta \Delta v_{\alpha}=-\alpha \Delta \partial_{t} u_{\alpha}-\beta \Delta u_{\alpha}+\partial_{t} f_{\alpha} \quad \text { a.e. in } Q \\
\partial_{n} v_{\alpha}=0 \quad \text { a.e. on } \Gamma \times(0, T) \\
v_{\alpha}(0)=v_{0, \alpha}+u_{0, \alpha}, \quad \partial_{t} v_{\alpha}(0)=\alpha \Delta v_{0, \alpha}+\beta \Delta w_{0, \alpha}+f_{\alpha}(0) \quad \text { a.e. in } \Omega .
\end{gathered}
$$

Due to (5.1) and (2.38), the right-hand side in (5.2) has exactly the same regularity here as its counterpart in (4.3) within the framework of Theorem 2.3. The same correspondence holds for the initial data in (5.4) and the related ones in (2.14): indeed, $v_{0, \alpha}+u_{0, \alpha}$ is in $W$ and $\alpha \Delta v_{0, \alpha}+\beta \Delta w_{0, \alpha}+f_{\alpha}(0)$ lies in $V$ due to (2.37) and (2.39). Then, we have that (cf. (4.1))

$$
v_{\alpha} \in W^{2,1}(0, T ; H) \cap W^{1, \infty}(0, T ; V) \cap H^{1}(0, T ; W)
$$

and $v_{\alpha}$ actually satisfies (5.2)-(5.4). Then, we are allowed to test (5.2) by $-\Delta \partial_{t} v_{\alpha}$ and repeat the computations developed in the Fourth a priori estimate. Thanks to (2.37) and (2.40), proceeding in this way we obtain (cf. (4.9))

$$
\begin{array}{r}
\frac{1}{2}\left\|\nabla \partial_{t} v_{\alpha}(t)\right\|_{H}^{2}+\frac{\alpha}{2} \int_{0}^{t}\left\|\Delta \partial_{t} v_{\alpha}(s)\right\|_{H}^{2} d s+\frac{\beta}{4}\left\|\Delta v_{\alpha}(t)\right\|_{H}^{2} \leq c_{\beta}+c \int_{0}^{t}\left\|\Delta v_{\alpha}(s)\right\|_{H}^{2} d s \\
+\int_{0}^{t}\left\|\partial_{t}^{2} f_{\alpha}^{(1)}(s)\right\|_{H}\left\|\Delta v_{\alpha}(s)\right\|_{H} d s+\int_{0}^{t}\left\|\nabla \partial_{t} f_{\alpha}^{(2)}(s)\right\|_{H}\left\|\nabla \partial_{t} v_{\alpha}(s)\right\|_{H} d s .
\end{array}
$$

By means of the generalised Gronwall lemma, stated in [2, Teorema 2.1, p. 245], recalling (2.38), (2.40) and $v_{\alpha}=\partial_{t} y_{\alpha}$, we can conclude

$$
\left\|\partial_{t} y_{\alpha}\right\|_{W^{1, \infty}(0, T ; V) \cap L^{\infty}(0, T ; W)}+\sqrt{\alpha}\left\|\partial_{t} y_{\alpha}\right\|_{H^{1}(0, T ; W)} \leq c_{\beta}
$$

which entails (2.41).

Second error estimate. We begin with revisiting the first estimate on the convergence error. Assume all the hypotheses of Theorem 2.9, and reconsider the proof of Theorem 2.4 we have given in Section 4. We leave unchanged all the estimates we have already established, except the inequalities (4.19): indeed, from (4.6) and (5.5) it follows that

$$
\begin{aligned}
\alpha \int_{Q t}\left(\Delta \partial_{t} y_{\alpha}-\Delta u_{\alpha}\right) \partial_{t} \widehat{y}_{\alpha} & \leq \frac{\alpha^{2}}{2} \int_{0}^{t}\left\|\Delta \partial_{t} y_{\alpha}(s)\right\|_{H}^{2} d s+\frac{\alpha^{2}}{2} \int_{0}^{t}\left\|\Delta u_{\alpha}(s)\right\|_{H}^{2} d s \\
& +\int_{0}^{t}\left\|\partial_{t} \widehat{y}_{\alpha}(s)\right\|_{H}^{2} d s \leq c_{\beta} \alpha^{2}+\int_{0}^{t}\left\|\partial_{t} \widehat{y}_{\alpha}(s)\right\|_{H}^{2} d s .
\end{aligned}
$$

By this new estimate and the assumptions (2.42)-(2.43), inequality (4.23) now becomes

$$
\begin{aligned}
& \frac{1}{2}\left\|\widehat{u}_{\alpha}(t)\right\|_{H}^{2}+\int_{0}^{t}\left\|\nabla \widehat{u}_{\alpha}(s)\right\|_{H}^{2} d s+\frac{1}{2}\left\|\partial_{t} \widehat{y}_{\alpha}(t)\right\|_{H}^{2}+\frac{\beta}{4}\left\|\widehat{y}_{\alpha}(t)\right\|_{V}^{2} \\
& \leq c_{\beta} \alpha^{2}+c \int_{0}^{t}\left\|\widehat{u}_{\alpha}(s)\right\|_{H}^{2} d s+c \int_{0}^{t}\left\|\partial_{t} \widehat{y}_{\alpha}(s)\right\|_{H}^{2} d s+c \int_{0}^{t}\left\|\nabla \widehat{y}_{\alpha}(s)\right\|_{H}^{2} d s \\
& \quad+\int_{0}^{t}\left\|\partial_{t} \widehat{f}_{\alpha}^{(1)}(s)\right\|_{V^{\prime}}\left\|\widehat{y}_{\alpha}(s)\right\|_{V} d s+\int_{0}^{t}\left\|\widehat{f}_{\alpha}^{(2)}(s)\right\|_{H}\left\|\partial_{t} \widehat{y}_{\alpha}(s)\right\|_{H} d s
\end{aligned}
$$


so that the generalised Gronwall lemma (cf., e.g., [2]) and (2.42) entail

$$
\left\|\widehat{y}_{\alpha}\right\|_{W^{1, \infty}(0, T ; H) \cap L^{\infty}(0, T ; V)}+\left\|\widehat{u}_{\alpha}\right\|_{L^{\infty}(0, T ; H) \cap L^{2}(0, T ; V)} \leq c_{\beta} \alpha .
$$

Next, we consider the equation (4.13), where we have set $\widehat{\xi}_{\alpha}=0$, and apply Lemma 3.1. by (2.43), the Lipschitz-continuity of $g$ and (5.6) we obtain

$$
\left\|\widehat{u}_{\alpha}\right\|_{H^{1}(0, T ; H) \cap L^{\infty}(0, T ; V) \cap L^{2}(0, T ; W)} \leq c\left\{\left\|\widehat{u}_{0, \alpha}\right\|_{V}+\left\|\widehat{u}_{\alpha}\right\|_{L^{2}(Q)}+\left\|\partial_{t} \widehat{y}_{\alpha}\right\|_{L^{2}(Q)}\right\} \leq c_{\beta} \alpha .
$$

Then (2.44) is entirely proved. In order to complete the proof of Theorem 2.9, we test equation (4.12) with $-\Delta \partial_{t} y_{\alpha}$ (which belongs to $L^{2}(Q)$ because of the estimate (5.5)) and integrate over $Q_{t}$ :

$$
\begin{aligned}
& \frac{1}{2}\left\|\nabla \partial_{t} \widehat{y}_{\alpha}(t)\right\|_{H}^{2}+\frac{\beta}{2}\left\|\Delta \widehat{y}_{\alpha}(t)\right\|_{H}^{2} \leq \frac{1}{2}\left\|\nabla\left(\widehat{v}_{0, \alpha}+\widehat{u}_{0, \alpha}\right)\right\|_{H}^{2}+\frac{\beta}{2}\left\|\Delta \widehat{w}_{0, \alpha}\right\|_{H}^{2} \\
& +\alpha \int_{Q_{t}}\left(\Delta u_{\alpha}-\Delta \partial_{t} y_{\alpha}\right) \Delta \partial_{t} \widehat{y}_{\alpha}+\beta \int_{Q_{t}} \Delta\left(1 * \widehat{u}_{\alpha}\right) \Delta \partial_{t} \widehat{y}_{\alpha}-\int_{Q_{t}} \widehat{f}_{\alpha} \Delta \partial_{t} \widehat{y}_{\alpha} .
\end{aligned}
$$

We deal with the integral involving $u_{\alpha}$ and $y_{\alpha}$, which is delicate to treat. By an application of the Hölder inequality, (4.24), and (5.5) - which holds for $y$ as well - we have

$$
\begin{aligned}
& \alpha \int_{Q_{t}}\left(\Delta u_{\alpha}-\Delta \partial_{t} y_{\alpha}\right) \Delta \partial_{t} \widehat{y}_{\alpha} \\
& \leq \alpha\left(\left\|\Delta u_{\alpha}\right\|_{L^{2}(Q)}+\left\|\Delta \partial_{t} y_{\alpha}\right\|_{L^{2}(Q)}\right)\left(\left\|\Delta \partial_{t} y_{\alpha}\right\|_{L^{2}(Q)}+\left\|\Delta \partial_{t} y\right\|_{L^{2}(Q)}\right) \leq c_{\beta} \alpha .
\end{aligned}
$$

The subsequent term is estimated by integrating by parts, with respect to time, and taking advantage of (3.2):

$$
\begin{gathered}
\beta \int_{Q_{t}} \Delta\left(1 * \widehat{u}_{\alpha}\right) \Delta \partial_{t} \widehat{y}_{\alpha} \leq 2 \beta\left\|\Delta\left(1 * \widehat{u}_{\alpha}\right)(t)\right\|_{H}^{2}+\frac{\beta}{8}\left\|\Delta \widehat{y}_{\alpha}(t)\right\|_{H}^{2}-\beta \int_{Q_{t}} \Delta \widehat{u}_{\alpha} \Delta \widehat{y}_{\alpha} \\
\leq \beta(4 T+1) \int_{0}^{t}\left\|\Delta \widehat{u}_{\alpha}(s)\right\|_{H}^{2} d s+\frac{\beta}{8}\left\|\Delta \widehat{y}_{\alpha}(t)\right\|_{H}^{2}+\frac{\beta}{4} \int_{0}^{t}\left\|\Delta \widehat{y}_{\alpha}(s)\right\|_{H}^{2} d s .
\end{gathered}
$$

Now we split $\widehat{f}_{\alpha}=\widehat{f}_{\alpha}^{(1)}+\widehat{f}_{\alpha}^{(2)}$, as usual, and deal on the respective terms separately, integrating by parts the former in time and the latter in space. We obtain

$$
\begin{aligned}
\int_{Q_{t}} \widehat{f}_{\alpha}^{(1)} \Delta \partial_{t} \widehat{y}_{\alpha} \leq & \frac{2}{\beta}\left\|\widehat{f}_{\alpha}^{(1)}(t)\right\|_{H}^{2}+\frac{\beta}{8}\left\|\Delta \widehat{y}_{\alpha}(t)\right\|_{H}^{2}+\frac{1}{2}\left\|\widehat{f}_{\alpha}^{(1)}(0)\right\|_{H}^{2} \\
& +\frac{1}{2}\left\|\Delta w_{0, \alpha}\right\|_{H}^{2}+\int_{0}^{t}\left\|\widehat{f}_{\alpha}^{(1)}(s)\right\|_{H}\left\|\Delta \widehat{y}_{\alpha}(s)\right\|_{H} d s
\end{aligned}
$$

and

$$
\int_{Q_{t}} \widehat{f}_{\alpha}^{(2)} \Delta \partial_{t} \widehat{y}_{\alpha} \leq \int_{0}^{t}\left\|\nabla \widehat{f}_{\alpha}^{(2)}(s)\right\|_{H}\left\|\nabla \partial_{t} \widehat{y}_{\alpha}(s)\right\|_{H} d s .
$$

According to all these estimates, and taking (2.45), (5.7) and (2.1) into account, the inequality (5.8) transforms into

$$
\begin{aligned}
& \frac{1}{2}\left\|\nabla \partial_{t} \widehat{y}_{\alpha}(t)\right\|_{H}^{2}+\frac{\beta}{4}\left\|\Delta \widehat{y}_{\alpha}(t)\right\|_{H}^{2} \leq c_{\beta} \alpha+\frac{\beta}{2} \int_{0}^{t}\left\|\Delta \widehat{y}_{\alpha}(s)\right\|_{H}^{2} d s \\
& \quad+\int_{0}^{t}\left\|\widehat{f}_{\alpha}^{(1)}(s)\right\|_{H}\left\|\Delta \widehat{y}_{\alpha}(s)\right\|_{H} d s+\int_{0}^{t}\left\|\nabla \widehat{f}_{\alpha}^{(2)}(s)\right\|_{H}\left\|\nabla \partial_{t} \widehat{y}_{\alpha}(s)\right\|_{H} d s .
\end{aligned}
$$


In view of this inequality and (2.45), by the generalised Gronwall lemma (cf., e.g., [2]) we deduce (2.46) and therefore conclude the proof of Theorem 2.9.

\section{References}

[1] J. Aguirre-Puente and M. Frémond, Frost propagation in wet porous media, in "Applications of methods of functional analysis to problems in mechanics" (Joint Sympos., IUTAM/IMU, Marseille, 1975), pp. 137-147. Lecture Notes in Math., 503. Springer, Berlin, 1976.

[2] C. Baiocchi, Sulle equazioni differenziali astratte lineari del primo e del secondo ordine negli spazi di Hilbert, Ann. Mat. Pura Appl., 76(4) (1967), 233-304.

[3] V. Barbu, "Nonlinear semigroups and differential equations in Banach spaces", Noordhoff, Leyden, 1976.

[4] H. Brezis, "Opérateurs maximaux monotones et semi-groupes de contractions dans les espaces de Hilbert", North-Holland Math. Stud. 5, North-Holland, Amsterdam,

[5] G. Caginalp, An analysis of a phase field model of a free boundary, Arch. Rational Mech. Anal. 92 (1986) 205-245.

[6] G. Caginalp and Y. Nishiura, The existence of travelling waves for phase field equations and convergence to sharp interface models in the singular limit, Quart. Appl. Math. 49 (1991) 147-162.

[7] G. Canevari and P. Colli, Solvability and asymptotic analisys of a generalization of the Caginalp phase field system, Commun. Pure Appl. Anal. 11 (2012) 1959-1982.

[8] P. Colli, G. Gilardi, and M. Grasselli, Global smooth solution to the standard phase field model with memory, Adv. Differential Equations 2 (1997) 453-486.

[9] P. Colli, G. Gilardi, and M. Grasselli, Well-posedness of the weak formulation for the phase-field model with memory, Adv. Differential Equations 2 (1997) 487-508.

[10] P. Colli and V. Recupero, Convergence to the Stefan problem of the phase relaxation problem with Cattaneo heat flux law, J. Evol. Equ. 2 (2002) 177-195.

[11] G. Duvaut, Résolution d'un problème de Stefan (fusion d'un bloc de glace à zéro degré), C. R. Acad. Sci. Paris Sér. A-B 276 (1973) A1461-A1463.

[12] M. Frémond, "Non-smooth Thermomechanics", Springer-Verlag, Berlin, 2002.

[13] A.E. Green and P.M. Naghdi, A re-examination of the basic postulates of thermomechanics, Proc. Roy. Soc. Lond. A 432 (1991) 171-194.

[14] A.E. Green and P.M. Naghdi, On undamped heat waves in an elastic solid, J. Thermal Stresses 15 (1992) 253-264.

[15] A.E. Green and P.M. Naghdi, Thermoelasticity without energy dissipation, J. Elasticity 31 (1993) 189-208. 
[16] A.E. Green and P.M. Naghdi, A new thermoviscous theory for fluids, J. NonNewtonian Fluid Mech. 56 (1995) 289-306.

[17] E. Magenes, Two-phase Stefan problems in several space variables, Matematiche (Catania) 36 (1981) 65-108 (1983).

[18] A. Miranville and R. Quintanilla, A generalization of the Caginalp phase-field system based on the Cattaneo law, Nonlinear Anal. 71 (2009) 2278-2290.

[19] A. Miranville and R. Quintanilla, Some generalizations of the Caginalp phase-field system, Appl. Anal. 88 (2009) 877-894.

[20] A. Miranville and R. Quintanilla, A Caginalp phase-field system with a nonlinear coupling, Nonlinear Anal. Real World Appl. 11 (2010) 2849-2861.

[21] A. Miranville and R. Quintanilla, A type III phase-field system with a logarithmic potential, Appl. Math. Lett. 24 (2011) 1003-1008.

[22] A. Miranville and R. Quintanilla, On a phase-field system based on the Cattaneo law, Nonlinear Anal. 75 (2012) 2552-2565

[23] J. Simon, Compact sets in the space $L^{p}(0, T ; B)$, Ann. Mat. Pura Appl. 146 (1987) 65-96. 